Article

\title{
A 4th-Order Optimal Extension of Ostrowski's Method for Multiple Zeros of Univariate Nonlinear Functions
}

\author{
Ramandeep Behl *,† and Waleed M. Al-Hamdan ${ }^{\dagger}$ \\ Department of Mathematics, King Abdulaziz University, Jeddah 21589, Saudi Arabia \\ * Correspondence: ramanbehl87@yahoo.in \\ † These authors contributed equally to this work.
}

Received: 27 June 2019 ; Accepted: 12 August 2019; Published: 1 September 2019

\begin{abstract}
We present a new optimal class of Ostrowski's method for obtaining multiple zeros of univariate nonlinear functions. Several researchers tried to construct an optimal family of Ostrowski's method for multiple zeros, but they did not have success in this direction. The new strategy adopts a weight function approach. The design structure of new families of Ostrowski's technique is simpler than the existing classical families of the same order for multiple zeros. The classical Ostrowski's method of fourth-order can obtain a particular form for the simple root. Their efficiency is checked on a good number of relevant numerical examples. These results demonstrate the performance of our methods. We find that the new methods are just as competent as other existing robust techniques available in the literature.
\end{abstract}

Keywords: multiple roots; nonlinear equations; Ostrowski's method; optimal convergence order; efficiency index

\section{Introduction}

Mathematics is always changing, and the way we teach it also changes as was presented in [1,2]. Moreover, in advanced mathematics, we need to use different alternatives since we all know the different problems that students present. In this paper, we present a study on iterative methods that can be used in postgraduate studies in order to teach them. Many problems of numerous disciplines such as applied sciences, scientific computing, and engineering [3-7] can be converted to mathematical expressions. Some of them can be transformed to an equation of the following form:

$$
\Delta(x)=0
$$

where $\Delta: \mathbb{D} \subseteq \mathbb{C} \rightarrow \mathbb{C}$ is an analytic map [8,9] in the region $\mathbb{D}$. It is almost inconceivable to retrieve the solution through an analytical method for every nonlinear univariate function except some of them. Therefore, we investigate the iterative schemes to attain an approximate solution of (1). We have several one- and multi-point iterative techniques that handle such problems. However, the problem with the one-point iterative schemes is the restrictions on the considered function. That is to say, whenever we want to increase the convergence order of a one-point iterative scheme, then we have to introduce some high-order derivation of $f$. For example, we need three functional values (i.e., $f, f^{\prime}$, and $f^{\prime \prime}$ ) in order to obtain the third-order convergence of an iterative method, and for fourth-order convergence, we require four functional values (i.e., $f, f^{\prime}, f^{\prime \prime}$, and $f^{\prime \prime \prime}$ ) (please have a look at [10] for more details).

Therefore, special attention has been paid to yielding optimal multi-point schemes that do not make obligatory the value of the second- or high-order derivation. Additionally, it is very 
vital and fascinating from the practical and computational point of view. The classical Kung-Traub conjecture [11] concluded that the convergence order of any multi-point technique without memory using $n$ functional evaluations each iteration cannot exceed the maximum bound $2^{n-1}$, known as the optimal order. Therefore, the optimal convergence order for an iterative scheme requiring three functional evaluations per iteration would be four.

One of the renowned and efficient multi-point iterative techniques for solving (1) is Ostrowski's [10,12]. This method has biquadratic convergence and is given by:

$$
\left\{\begin{array}{c}
y_{l}=x_{l}-\frac{\Delta\left(x_{l}\right)}{\Delta^{\prime}\left(x_{l}\right)} \\
x_{l+1}=x_{l}-\frac{\Delta\left(x_{l}\right)}{\Delta^{\prime}\left(x_{l}\right)}\left[\frac{\Delta\left(x_{l}\right)-\Delta\left(y_{l}\right)}{\Delta\left(x_{l}\right)-2 \Delta\left(y_{l}\right)}\right] .
\end{array}\right.
$$

Ostrowski's method approximates the root of a nonlinear equation in one variable using two values of $\Delta$ and one of its first-order derivation $\Delta^{\prime}$. Therefore, this is an optimal method by the Kung-Traub conjecture [11]. However, this method converges linearly for multiple roots.

The construction of optimal multi-point techniques for multiple zeros of (1) is another important and difficult problem. Some high-order multi-point schemes for multiple zeros were suggested by Dong [13,14], Kumar et al. [15], Neta et al. [16-19], and Li et al. [20,21]. Some of them are of third-order [13,14,16,18], while others have fourth-order convergence [17,19-21].

Li et al. [20,21] suggested some fourth-order techniques based on Jarratt's method [22]. However, only two of them were optimal and given as follows:

$$
\left\{\begin{array}{c}
y_{l}=x_{l}-\frac{2 m}{m+2} \frac{\Delta\left(x_{l}\right)}{\Delta^{\prime}\left(x_{l}\right)^{\prime}} \\
x_{l+1}=x_{l}-\frac{m \Delta\left(x_{l}\right)}{4\left(\frac{m}{m+2}\right)^{m-1} \Delta^{\prime}\left(x_{l}\right)}\left[\frac{m^{3}(m-2) \Delta^{\prime 2}\left(y_{l}\right)-\left(\frac{m}{m+2}\right)^{2(m-1)}\left(m^{4}+2 m^{3}+4 m^{2}+8 m+16\right) \Delta^{\prime 2}\left(x_{l}\right)}{\left(\frac{m}{m+2}\right)^{m-1}\left(m^{3}+3 m^{2}+2 m-4\right) \Delta^{\prime 2}\left(x_{l}\right)-m^{2}(m+3) \Delta^{\prime}\left(x_{l}\right) \Delta^{\prime}\left(y_{l}\right)}\right],
\end{array}\right.
$$

and:

$$
\left\{\begin{array}{c}
y_{l}=x_{l}-\frac{2 m}{m+2} \frac{\Delta\left(x_{l}\right)}{\Delta^{\prime}\left(x_{l}\right)} \\
x_{l+1}=x_{l}-b_{1} \frac{\Delta\left(x_{l}\right)}{\Delta^{\prime}\left(x_{l}\right)}-\frac{\Delta\left(x_{l}\right)}{b_{2} \Delta^{\prime}\left(x_{l}\right)+b_{3} \Delta^{\prime}\left(y_{l}\right)}
\end{array}\right.
$$

where $b_{1}=m-\frac{m^{2}}{2}, b_{2}=-\frac{1}{m}, b_{3}=\frac{1}{m\left(\frac{m}{m+2}\right)^{m}}$ and $m$ is the known multiplicity of the required zero of considered function $\Delta$.

Sharma and Sharma [23] introduced an optimal iterative scheme, defined as below:

$$
\left\{\begin{array}{c}
y_{l}=x_{l}-\frac{2 m}{m+2} \frac{\Delta\left(x_{l}\right)}{\Delta^{\prime}\left(x_{l}\right)} \\
x_{l+1}=x_{l}-a_{1} w_{1}\left(x_{l}\right)-a_{2} w_{2}\left(x_{l}\right)-a_{3} \frac{w_{2}^{2}\left(x_{l}\right)}{w_{1}\left(x_{l}\right)}
\end{array}\right.
$$

where $w_{1}\left(x_{l}\right)=\frac{\Delta\left(x_{l}\right)}{\Delta^{\prime}\left(x_{l}\right)}, w_{2}\left(x_{l}\right)=\frac{\Delta\left(x_{l}\right)}{\Delta^{\prime}\left(y_{l}\right)}, a_{1}=\frac{m}{8}\left(m^{3}-4 m-8\right), a_{2}=-\frac{m}{4}(m-1)(m+2)^{2}\left(\frac{m}{m+2}\right)^{m}$ and $a_{3}=\frac{m}{8}(m+2)^{3}\left(\frac{m}{m+2}\right)^{2 m}$.

More recently, Zhou et al. [24] established a family of fourth-order optimal methods with the help of the weight-function technique, which is given by:

$$
\left\{\begin{array}{c}
y_{l}=x_{l}-t \frac{\Delta\left(x_{l}\right)}{\Delta^{\prime}\left(x_{l}\right)}, \\
x_{l+1}=x_{l}-\frac{\Delta\left(x_{l}\right)}{\Delta^{\prime}\left(x_{l}\right)} H\left(\frac{\Delta^{\prime}\left(y_{l}\right)}{\Delta^{\prime}\left(x_{l}\right)}\right),
\end{array}\right.
$$


where the weight function $H(\cdot) \in \mathbb{C}^{2}(\mathbb{R})$. It is straightforward to see that all above-mentioned methods are the optimal variants of Jarratt's methods and have complicated body structures, except (4).

The construction of an optimal family of Ostrowski's scheme [10,12] for obtaining multiple zeros of (1) is a tough task in the field of numerical analysis. Recently, some attempts were made by Kumar et al. [15] on the development of an optimal class of Ostrowski's technique for obtaining multiple roots. However, they did not have any progress in this regard. Therefore, we have no optimal family of the fourth-order Ostrowski technique for obtaining multiple root of nonlinear equation numerically, till today.

With this aim, we are keen to suggest a family of the optimal Ostrowski method for multiple roots. The new strategy is based on the weight function approach. We offer families of iterative methods, which demonstrate more competent behavior as compared to the existing classical techniques available in the literature. Here, we adopt the programming package Mathematica9 for symbolic computation in order to attain the general error equation of iterative schemes.

\section{Construction of the Novel Technique}

Here, we suggest the following iterative scheme:

$$
\left\{\begin{array}{c}
y_{l}=x_{l}-m \frac{\Delta\left(x_{l}\right)}{\Delta^{\prime}\left(x_{l}\right)} \\
x_{l+1}=x_{l}-m \frac{\Delta\left(x_{l}\right)}{\Delta^{\prime}\left(x_{l}\right)}\left[\frac{1-\mu}{1-2 \mu}\right] Q(\mu),
\end{array}\right.
$$

where the weight function $Q(\mu): \mathbb{C} \rightarrow \mathbb{C}$ is an analytic/holomorphic map $[8,9]$ in the neighborhood of origin (0) with $\mu=\left(\frac{\Delta\left(y_{l}\right)}{\Delta\left(x_{l}\right)}\right)^{\frac{1}{m}}$. Since, the function $\mu$ is a multi-valued. Hence, we considered their principal analytic branches (the details can be seen in $[25,26])$. Additionally, it is labor-saving to treat $\mu$ as a principal root known as $\mu=\exp \left[\frac{1}{m} \log \left(\frac{\Delta\left(y_{l}\right)}{\Delta\left(x_{l}\right)}\right)\right]$, with $\log \left(\frac{\Delta\left(y_{l}\right)}{\Delta\left(x_{l}\right)}\right)=\log \left|\frac{\Delta\left(y_{l}\right)}{\Delta\left(x_{l}\right)}\right|+i \operatorname{Arg}\left(\frac{\Delta\left(y_{l}\right)}{\Delta\left(x_{l}\right)}\right)$ for $-\pi<\operatorname{Arg}\left(\frac{\Delta\left(y_{l}\right)}{\Delta\left(x_{l}\right)}\right) \leq \pi$; this convention of $\operatorname{Arg}(z)$ for $z \in \mathbb{C}$ agrees with that of $\log [z]$, to be employed later in Sections 2 and 4. Furthermore, we obtain that $\mu=\left|\frac{\Delta\left(y_{l}\right)}{\Delta\left(x_{l}\right)}\right|^{\frac{1}{m}} \cdot \exp \left[\frac{i}{m} \operatorname{Arg}\left(\frac{\Delta\left(y_{l}\right)}{\Delta\left(x_{l}\right)}\right)\right]=$ $O\left(e_{l}^{2}\right)$, by means of additional investigation of $\mu$.

Theorem 1 illustrates that the scheme (7) reaches a fourth-order convergence without adopting any supplementary functional value. The weight function $Q(\mu)$ plays a vital role in the development of the scheme (please see Theorem 1 for further details).

Theorem 1. Let us assume $\Delta: \mathbb{D} \subseteq \mathbb{C} \rightarrow \mathbb{C}$ is a holomorphic function in the region $\mathbb{D}$, enclosing the required multiple zero (say $x=r_{m}$ ) of $\Delta(x)$ having known multiplicity $m \geq 1$. Then, the scheme (7) has fourth-order convergence if it employs:

$$
Q(0)=1, \quad Q^{\prime}(0)=0, \quad Q^{\prime \prime}(0)=0 .
$$

The scheme (7) gives the following error expression:

$$
e_{l+1}=\left(\frac{\left(3 m+3-Q^{\prime \prime \prime}(0)\right) c_{1}^{3}-6 m c_{1} c_{2}}{6 m^{3}}\right) e_{l}^{4}+O\left(e_{l}^{5}\right),
$$

where $Q^{\prime \prime \prime}(0) \in \mathbb{R}$, error at the $l^{\text {th }}$ step $e_{l}=x_{l}-r_{m}$, and $c_{k}=\frac{m !}{m+k !} \frac{\Delta^{(m+k)}\left(r_{m}\right)}{\Delta^{(m)}\left(r_{m}\right)}, k=1,2,3,4$. 
Proof. We assumed that $x=r_{m}$ is a multiple zero (with known multiplicity $m \geq 1$ ) of (1). We expanded $\Delta\left(x_{l}\right)$ and $\Delta^{\prime}\left(x_{l}\right)$ about a point $x=r_{m}$ by the Taylor's series expansion, leading to the following:

$$
\Delta\left(x_{l}\right)=\frac{f^{(m)}\left(r_{m}\right)}{m !} e_{l}^{m}\left(1+c_{1} e_{l}+c_{2} e_{l}^{2}+c_{3} e_{l}^{3}+c_{4} e_{l}^{4}+O\left(e_{l}^{5}\right)\right),
$$

and:

$$
\Delta^{\prime}\left(x_{l}\right)=\frac{f^{(m-1)}\left(r_{m}\right)}{(m-1) !} e_{l}^{m-1}\left(1+\frac{m+1}{m} c_{1} e_{l}+\frac{m+2}{m} c_{2} e_{l}^{2}+\frac{m+3}{m} c_{3} e_{l}^{3}+\frac{(m+4)}{m} c_{4} e_{l}^{4}+O\left(e_{l}^{5}\right)\right),
$$

respectively.

By adopting (10) and (11), we further yielded:

$$
\frac{\Delta\left(x_{l}\right)}{\Delta^{\prime}\left(x_{l}\right)}=\frac{1}{m} e_{l}-\frac{c_{1}}{m^{2}} e_{l}^{2}+\left(\frac{(m+1) c_{1}^{2}-2 m c_{2}}{m^{3}}\right) e_{l}^{3}+O\left(e_{l}^{4}\right),
$$

and:

$$
\begin{aligned}
\Delta\left(y_{l}\right)= & \Delta\left(x_{l}-m \frac{\Delta\left(x_{l}\right)}{\Delta^{\prime}\left(x_{l}\right)}\right), \\
= & \frac{\Delta^{(m)}\left(r_{m}\right)}{m !}\left(\frac{c_{1}}{m}\right)^{m} e_{l}^{2 m}\left[1+\left(\frac{\left(2 m c_{2}-(m+1) c_{1}^{2}\right)}{c_{1}}\right) e_{l}\right. \\
& \left.+\left(\frac{\left(m^{3}+3 m^{2}+3 m+3\right) c_{1}^{4}-2 m\left(2 m^{2}+3 m+2\right) c_{1}^{2} c_{2}+4 m^{2}(m-1) c_{2}^{2}+6 m^{2} c_{1} c_{3}}{2 c_{1}^{3}}\right) e_{l}^{2}+O\left(e_{l}^{3}\right)\right] .
\end{aligned}
$$

Using Expressions (10) and (13), we obtained:

$$
\begin{aligned}
\mu=\left(\frac{\Delta\left(y_{l}\right)}{\Delta\left(x_{l}\right)}\right)^{\frac{1}{m}} & =\left(\frac{c_{1}}{m}\right) e_{l}+\left(\frac{\left(2 m c_{2}-(m+2) c_{1}^{2}\right)}{m^{2}}\right) e_{l}^{2} \\
& +\left(\frac{\left(2 m^{2}+7 m+7\right) c_{1}^{3}-2 m(3 m+7) c_{1} c_{2}+6 m^{2} c_{3}}{2 m^{3}}\right) e_{l}^{3}+O\left(e_{l}^{4}\right) .
\end{aligned}
$$

and:

$$
\begin{aligned}
{\left[\frac{1-\mu}{1-2 \mu}\right] } & =1+\left(\frac{c_{1}}{m}\right) e_{l}-\left(\frac{c_{1}^{2}-2 c_{2}}{m}\right) e_{l}^{2}+\left(\frac{\left(2 m^{2}-m-1\right) c_{1}^{3}-2 m(3 m-1) c_{1} c_{2}+6 m^{2} c_{3}}{2 m^{3}}\right) e_{l}^{3} \\
& +O\left(e_{l}^{4}\right)
\end{aligned}
$$

We concluded from Expression (14) that $\mu=\left(\frac{\Delta\left(y_{l}\right)}{\Delta\left(x_{l}\right)}\right)^{\frac{1}{m}}$ is of linear order $e_{l}$. Therefore, we can expand $Q(\mu)$ in the neighborhood of origin (0) in the following way:

$$
\begin{aligned}
Q(\mu) & =Q(0)+\mu Q^{\prime}(0)+\frac{1}{2 !} \mu^{2} Q^{\prime \prime}(0)+\frac{1}{3 !} \mu^{3} Q^{\prime \prime \prime}(0)+O\left(e_{l}^{4}\right) \\
& =Q(0)+\frac{c_{1} Q^{\prime}(0)}{m} e_{l}+\frac{\left(c_{1}^{2}\left(Q^{\prime \prime}(0)-2(m+2) Q^{\prime}(0)\right)+4 c_{2} m Q^{\prime}(0)\right)}{2 m^{2}} e_{l}^{2} \\
& +\frac{c_{1}^{3}\left(3\left(2 m^{2}+7 m+7\right) Q^{\prime}(0)-6(m+2) Q^{\prime \prime}(0)+Q^{\prime \prime \prime}(0)\right)+18 c_{3} m^{2} Q^{\prime}(0)-6 c_{2} c_{1} m\left((3 m+7) Q^{\prime}(0)-2 Q^{\prime \prime}(0)\right)}{6 m^{3}} e_{l}^{3} \\
& +O\left(e_{l}^{4}\right),
\end{aligned}
$$

by adopting Taylor's series expansion. 
Using Equations (12), (15) and (16) in the technique (7), we have:

$$
\begin{aligned}
e_{l+1} & =e_{l}-m \frac{\Delta\left(x_{l}\right)}{\Delta^{\prime}\left(x_{l}\right)}\left[\frac{1-\mu}{1-2 \mu}\right] Q(\mu) \\
& =(1-Q(0)) e_{l}-\left(\frac{Q^{\prime}(0) c_{1}}{m}\right) e_{l}^{2}+\left(\frac{\left(2(m+2) Q^{\prime}(0)-Q^{\prime \prime}(0)\right) c_{1}^{2}-4 m Q^{\prime}(0) c_{2}}{2 m^{2}}\right) e_{l}^{3} \\
& +\frac{1}{6 m^{3}}\left[\left(3(m+1) Q(0)-3\left(2 m^{2}+7 m+7\right) Q^{\prime}(0)+6(m+2) Q^{\prime \prime}(0)-Q^{\prime \prime \prime}(0)\right) c_{1}^{3}-6 m(Q(0)\right. \\
& \left.\left.+2 Q^{\prime \prime}(0)-(3 m+7) Q(0)\right) c_{1} c_{2}-18 m^{2} Q^{\prime}(0) c_{3}\right] e_{l}^{4}+O\left(e_{l}^{5}\right) .
\end{aligned}
$$

The coefficients of $e_{l}, e_{l}^{2}$, and $e_{l}^{3}$ of Expression (17) should be zero at the same time for attaining fourth-order convergence. From Expression (17), we yield the following expressions that involve $Q(0), Q^{\prime}(0), Q^{\prime \prime}(0)$, and $Q^{\prime \prime \prime}(0)$ :

$$
\left\{\begin{array}{l}
-Q(0)+1=0 \\
Q^{\prime}(0)=0 \\
\left(2(m+2) Q^{\prime}(0)-Q^{\prime \prime}(0)\right)=0
\end{array}\right.
$$

which further produce:

$$
Q(0)=1, \quad Q^{\prime}(0)=0, \quad Q^{\prime \prime}(0)=0 .
$$

By adopting (19) in (17), we have the following asymptotic constant and error equation of Scheme (7):

$$
e_{l+1}=\left(\frac{\left(3 m+3-Q^{\prime \prime \prime}(0)\right) c_{1}^{3}-6 m c_{1} c_{2}}{6 m^{3}}\right) e_{l}^{4}+O\left(e_{l}^{5}\right)
$$

where $Q^{\prime \prime \prime}(0) \in \mathbb{R}$.

This confirms that the new scheme (7) attains the optimal convergence order by just consuming three functional evaluations at each step. Hence, it completes the proof.

\section{Particular Forms}

It is straightforward to see from Theorem 1 that by using some specific values of $Q^{\prime \prime \prime}(0)$ and by adopting different kinds of weight functions, one can get modified families of Ostrowski's method. Therefore, we also propose some special forms of (7) that depend on the weight function $Q(x)$.

Case 1. We assumed a polynomial weight function, which is defined as follows:

$$
Q(\mu)=A \mu^{3}+1,
$$

where $Q^{\prime \prime \prime}(0)=6 A, A \in \mathbb{R}$.

By using (21) in Scheme (7), we yielded a new optimal family of fourth-order iterative methods, which is defined by:

$$
\left\{\begin{aligned}
y_{l} & =x_{l}-m \frac{\Delta\left(x_{l}\right)}{\Delta^{\prime}\left(x_{l}\right)} \\
x_{l+1} & =x_{l}-m \frac{\Delta\left(x_{l}\right)}{\Delta^{\prime}\left(x_{l}\right)}\left[\frac{1-\mu}{1-2 \mu}\right]\left[A \mu^{3}+1\right] .
\end{aligned}\right.
$$

Some sub-special cases of (22): 
(i) For $A=0$, Scheme (22) reads as:

$$
\left\{\begin{array}{c}
y_{l}=x_{l}-m \frac{\Delta\left(x_{l}\right)}{\Delta^{\prime}\left(x_{l}\right)} \\
x_{l+1}=x_{l}-m \frac{\Delta\left(x_{l}\right)}{\Delta^{\prime}\left(x_{l}\right)}\left[\frac{1-\mu}{1-2 \mu}\right] .
\end{array}\right.
$$

It is a new fourth-order Ostrowski method for multiple roots. For $m=1$, we get the famous Ostrowski technique that works only for simple roots.

(ii) For $A=\frac{1}{10}$, the family (22) is provided as:

$$
\left\{\begin{aligned}
y_{l} & =x_{l}-m \frac{\Delta\left(x_{l}\right)}{\Delta^{\prime}\left(x_{l}\right)} \\
x_{l+1} & =x_{l}-\frac{m}{10} \frac{\Delta\left(x_{l}\right)}{\Delta^{\prime}\left(x_{l}\right)}\left[\frac{1-\mu}{1-2 \mu}\right]\left(\mu^{3}+10\right) .
\end{aligned}\right.
$$

The above Expression (24) is another new fourth-order scheme.

(iii) For $A=\frac{1}{100}$, the family (22) yields another new scheme in the following way:

$$
\left\{\begin{aligned}
y_{l} & =x_{l}-m \frac{\Delta\left(x_{l}\right)}{\Delta^{\prime}\left(x_{l}\right)} \\
x_{l+1} & =x_{l}-\frac{m}{100} \frac{\Delta\left(x_{l}\right)}{\Delta^{\prime}\left(x_{l}\right)}\left[\frac{1-\mu}{1-2 \mu}\right]\left(\mu^{3}+100\right) .
\end{aligned}\right.
$$

Case 2. Let us choose a rational weight function, which is given by:

$$
Q(\mu)=\frac{A \mu^{3}+\mu-1}{\mu-1}, \mu \neq 1,
$$

where $Q^{\prime \prime \prime}(0)=-6 A$ and $A$ is any finite real number. By assuming the above weight function (26) in Expression (7), we have:

$$
\left\{\begin{array}{c}
y_{l}=x_{l}-m \frac{\Delta\left(x_{l}\right)}{\Delta^{\prime}\left(x_{l}\right)} \\
x_{l+1}=x_{l}-m \frac{\Delta\left(x_{l}\right)}{\Delta^{\prime}\left(x_{l}\right)}\left(\frac{A \mu^{3}+\mu-1}{2 \mu-1}\right),
\end{array}\right.
$$

which is another new family of iterative methods.

Some sub-special cases of (27):

(i) For $A=2$, Scheme (27) reads as:

$$
\left\{\begin{array}{c}
y_{l}=x_{l}-m \frac{\Delta\left(x_{l}\right)}{\Delta^{\prime}\left(x_{l}\right)} \\
x_{l+1}=x_{l}-m \frac{\Delta\left(x_{l}\right)}{\Delta^{\prime}\left(x_{l}\right)}\left(\frac{2 \mu^{3}+\mu-1}{2 \mu-1}\right) .
\end{array}\right.
$$

In this way, we obtain another new fourth-order optimal iterative technique. 
(ii) For $A=4$, the family (27) leads us to:

$$
\left\{\begin{array}{c}
y_{l}=x_{l}-m \frac{\Delta\left(x_{l}\right)}{\Delta^{\prime}\left(x_{l}\right)} \\
x_{l+1}=x_{l}-m \frac{\Delta\left(x_{l}\right)}{\Delta^{\prime}\left(x_{l}\right)}\left(2 \mu^{2}+\mu+1\right)
\end{array}\right.
$$

a new optimal fourth-order iterative scheme.

Case 3. We picked another rational function that is defined by the following weight function:

$$
Q(\mu)=\frac{A}{A+4 \mu}+\frac{4 A \mu}{(A+2 \mu)^{2}},
$$

where $Q^{\prime \prime \prime}(0)=-\frac{96}{A^{3}}, A \neq 0 \in \mathbb{R}$.

By adopting (30) in Expression (7), we obtained the following new optimal family of fourth-order methods:

$$
\left\{\begin{array}{c}
y_{l}=x_{l}-m \frac{\Delta\left(x_{l}\right)}{\Delta^{\prime}\left(x_{l}\right)} \\
x_{l+1}=x_{l}-m \frac{\Delta\left(x_{l}\right)}{\Delta^{\prime}\left(x_{l}\right)}\left[\frac{1-\mu}{1-2 \mu}\right]\left[\frac{A}{A+4 \mu}+\frac{4 A \mu}{(A+2 \mu)^{2}}\right] .
\end{array}\right.
$$

Some sub-special cases of Scheme (31):

(i) For $A=-4$, family the (31) provides us the special case of (31):

$$
\left\{\begin{array}{c}
y_{l}=x_{l}-m \frac{\Delta\left(x_{l}\right)}{\Delta^{\prime}\left(x_{l}\right)} \\
x_{l+1}=x_{l}-m \frac{\Delta\left(x_{l}\right)}{\Delta^{\prime}\left(x_{l}\right)}\left[\frac{-5 \mu^{2}+8 \mu-4}{(\mu-2)^{2}(2 \mu-1)}\right] .
\end{array}\right.
$$

(ii) For $A=-2$, Family (31) reads as:

$$
\left\{\begin{array}{c}
y_{l}=x_{l}-m \frac{\Delta\left(x_{l}\right)}{\Delta^{\prime}\left(x_{l}\right)} \\
x_{l+1}=x_{l}-m \frac{\Delta\left(x_{l}\right)}{\Delta^{\prime}\left(x_{l}\right)}\left[\frac{-5 \mu^{2}+4 \mu-1}{(1-2 \mu)^{2}(\mu-1)}\right],
\end{array}\right.
$$

another new fourth-order optimal iterative method.

Remark 1. The prominent character of our manuscript is that we proposed an optimal family of Ostrowski's technique for obtaining multiple zeros of univariate nonlinear functions, which was not accessible in the literature, to date.

Remark 2. It is straightforward to see that the body structure of our scheme is simpler than the existing methods available in the literature.

Remark 3. For $A=0$ and $m=1$ in Expression (22), the classical existing Ostrowski method for the simple root is a special form of new suggested family (7). In the majority of the numerical examples for simple roots, Ostrowski's method converges faster to the required root as compared to the exiting fourth-order iterative methods. Therefore, if we choose any real number close to zero, then the numerical results of the new methods will also be comparable to the classical Ostrowski method. This is one of the reasons to choose the values of A close to zero. Perhaps, someone could obtain different numerical results by choosing distinct numerical examples with the 
same methods (24) and (25). The reason behind this is the convergence of iterative methods, which depended on several things, like the initial approximation, the considered function, the required zero, etc.

Remark 4. It is vital to note that (7) consumes only three new functional values $\Delta\left(x_{l}\right), \Delta^{\prime}\left(x_{l}\right)$ and $\Delta\left(y_{l}\right)$, at each iteration. Therefore, Theorem 1 shows that the new scheme (7) has an optimal fourth-order convergence, as predicted by the Kung-Traub conjecture [11].

Remark 5. It is straightforward to see from the families, namely (22), (27) and (31), that one can construct several new fourth-order methods by adopting some specific values for disposable parameter $A$.

Remark 6. Finally, we concluded that by choosing distinct weight functions in Scheme (7), one can easily propose many new fourth-order optimal families of Ostrowski's technique.

\section{Numerical Results}

We illustrate the effectiveness of our optimal methods in this section. We used the new methods, namely Methods (23)-(25) denoted as (MOM1), (MOM2), and (MOM3), respectively, to solve the nonlinear equations depicted in Table 1. We computed them with the fourth-order optimal techniques, namely Expressions (3)-(5) and Method (11) from Zhou et al. [24], which is given by:

$$
\left\{\begin{aligned}
y_{l}= & x_{l}-\frac{2 m}{m+2} \frac{\Delta\left(x_{l}\right)}{\Delta^{\prime}\left(x_{l}\right)} \\
x_{l+1}= & x_{l}-\frac{m}{8}\left[m^{3}\left(\frac{m+2}{m}\right)^{2 m}\left(\frac{\Delta^{\prime}\left(y_{l}\right)}{\Delta^{\prime}\left(x_{l}\right)}\right)^{2}-2 m^{2}(m+3)\left(\frac{m+2}{m}\right)^{m} \frac{\Delta^{\prime}\left(y_{l}\right)}{\Delta^{\prime}\left(x_{l}\right)}\right. \\
& \left.+\left(m^{3}+6 m^{2}+8 m+8\right)\right] \frac{\Delta\left(x_{l}\right)}{\Delta^{\prime}\left(x_{l}\right)}
\end{aligned}\right.
$$

denoted by (LS1), (LS2), (SAS), and (Zhou 11), respectively. We demonstrate the comparisons of all the above-mentioned techniques in Table 2.

Table 1. A collection of nonlinear univariate functions.

\begin{tabular}{lll}
\hline$\Delta(x)$ & $\boldsymbol{r}_{\boldsymbol{m}}$ & $\mathbf{m}$ \\
\hline$\Delta_{1}(x)=\left(\sin ^{2} x-x^{2}+1\right)^{2}$ & 1.404491648215341 & 2 \\
$\Delta_{2}(x)=(x-5)^{3}$ & 5 & 3 \\
$\Delta_{3}(x)=\left(e^{x^{2}+7 x-30}-1\right)^{4}$ & 3 & 4 \\
$\Delta_{4}(x)=\left((x-1)^{3}-1\right)^{6}$ & 2 & 6 \\
$\Delta_{5}(x)=\left(e^{x}+x-20\right)^{2}$ & 2.842438953784447 & 2 \\
$\Delta_{6}(x)=(\cos x-x)^{4}$ & 0.739085133215161 & 4 \\
$\Delta_{7}(x)=\left(x^{2}-e^{x}-3 x+2\right)^{3}$ & 0.257530285439860 & 3 \\
$\Delta_{8}(x)=\left(x^{2}-16\right)^{3}$ & 4 & 3 \\
$\Delta_{9}(x)=\left(x^{3}-12 x^{2}+44 x-48\right)^{3}$ & 2 & 3 \\
$\Delta_{10}(x)=x^{3} \sin 4 x$ & 0 & 4 \\
\hline
\end{tabular}

We used the programming package Mathematica9 for symbolic computation in order to attain the general error equation of iterative schemes. On the other hand, the comparisons of iterative methods based on numerical examples were executed by adopting MATLAB Version 7.5 (R2007b) in double precision arithmetic. The stopping criteria are defined as follows:

(i) $\left|x_{l+1}-x_{l}\right|<\epsilon$,

(ii) $\left|\Delta\left(x_{l}\right)\right|<\epsilon$,

where $\epsilon=10^{-15}$ is a tolerance error. 
For the notation $(a, b)$ in Table 2, $a$ denotes the number of iterations and $b$ the total number of functional evaluations.

Table 2. Comparison of different iterative techniques.

\begin{tabular}{ccccccccc}
\hline$\Delta(x)$ & Initial & LS1 & LS2 & SAS & Zhou 11 & MOM1 & MOM2 & MOM3 \\
\hline 1. & 1.2 & $(4,12)$ & $(4,12)$ & $(3,9)$ & $(5,15)$ & $(4,12)$ & $(3,9)$ & $(4,12)$ \\
& 2.5 & $(3,9)$ & $(3,9)$ & $(3,9)$ & $(3,9)$ & $(3,9)$ & $(3,9)$ & $(3,9)$ \\
2. & 5.5 & $(3,9)$ & $(3,9)$ & $(3,9)$ & $(3,9)$ & $(1,3)$ & $(1,3)$ & $(1,3)$ \\
& 6.5 & $(2,6)$ & $(3,9)$ & $(2,6)$ & $(2,6)$ & $(1,3)$ & $(1,3)$ & $(1,3)$ \\
3. & 3.25 & $(4,12)$ & $(6,18)$ & $(9,27)$ & $(7,21)$ & $(4,12)$ & $(4,12)$ & $(4,12)$ \\
& 4.25 & $(12,36)$ & $(12,36)$ & $(14,42)$ & $(12,36)$ & $(10,30)$ & $(9,27)$ & $(10,30)$ \\
4. & 1.5 & CUR & CUR & $(9,27)$ & $(7,21)$ & $(3,9)$ & $(8,24)$ & $(7,21)$ \\
& 3.0 & $(4,12)$ & $(4,12)$ & $(5,15)$ & $(6,18)$ & $(3,9)$ & $(4,12)$ & $(3,9)$ \\
5. & 2.7 & $(3,9)$ & $(3,9)$ & $(3,9)$ & $(4,12)$ & $(3,9)$ & $(3,9)$ & $(3,9)$ \\
& 3.0 & $(3,9)$ & $(3,9)$ & $(3,9)$ & $(3,9)$ & $(2,6)$ & $(3,9)$ & $(3,9)$ \\
6. & 0.5 & $(3,9)$ & $(3,9)$ & $(3,9)$ & $(3,9)$ & $(3,9)$ & $(3,9)$ & $(3,9)$ \\
& 1.5 & $(3,9)$ & $(3,9)$ & $(3,9)$ & $(4,12)$ & $(3,9)$ & $(3,9)$ & $(3,9)$ \\
7. & -0.5 & $(3,9)$ & $(5,15)$ & $(4,12)$ & $(4,12)$ & $(3,9)$ & $(3,9)$ & $(3,9)$ \\
& 1 & $(3,9)$ & $(3,9)$ & $(3,9)$ & $(3,9)$ & $(2,6)$ & $(2,6)$ & $(2,6)$ \\
8. & 3.6 & $(5,15)$ & $(8,24)$ & $(8,24)$ & $(7,21)$ & $(4,12)$ & $(4,12)$ & $(4,12)$ \\
& 4.6 & $(2,6)$ & $(3,9)$ & $(2,6)$ & $(3,9)$ & $(2,6)$ & $(2,6)$ & $(2,6)$ \\
9. & 1 & $(5,15)$ & $(3,9)$ & $(6,18)$ & $(4,12)$ & $(3,9)$ & $(3,9)$ & $(3,9)$ \\
& 2.55 & CUR & CUR & $(10,30)$ & $(9,27)$ & $(6,18)$ & CUR & $(5,15)$ \\
10. & -1 & D & D & D & D & $(5,15)$ & $(5,15)$ & $(5,15)$ \\
& 1 & D & D & D & D & $(5,15)$ & $(5,15)$ & $(5,15)$ \\
\hline
\end{tabular}

"CUR" and "D" means that the methods converged to an undesired solution and diverged to the corresponding text functions, respectively. Our Methods (23)-(25) were superior to the techniques (4) and (5). From Table 2, we can see that our proposed methods can compete with the other methods, namely Methods (3)-(6) and require fewer iterative steps to reach the desired accuracy of the root.

\section{Concluding Remarks}

In this investigation, we constructed new optimal variants of Ostrowski's method for computing the multiple zeros $(m \geq 1)$ of univariate nonlinear functions. Our Scheme (7) used only two values of the function and one of its first-order derivatives at each iteration step. The new methods did not use the values of the second- or high-order derivation. The main advantage of the new scheme is the weight function $Q(\mu)$. By considering new weight functions employing the conditions (8), we could easily obtain several new fourth-order optimal iterative methods. Some of the new methods were mentioned in the numerical section. Finally, we concluded from the numerical experimentation that the new methods had at a minimum equal execution in comparison with other similar methods having the same convergence order. Someone may obtain different results on distinct numerical examples.

Author Contributions: All the authors gave equal contribution.

Funding: This research received no external funding.

Conflicts of Interest: The authors declare no conflict of interest.

\section{References}

1. Tello, J.I.C.; Orcos, L.; Granados, J.J.R. Virtual forums as a learning method in Industrial Engineering Organization. IEEE Lat. Am. Trans. 2016, 14, 3023-3028. [CrossRef]

2. LeTendre, G.; McGinnis, E.; Mitra, D.; Montgomery, R.; Pendola, A. The American Journal of Education: Challenges and opportunities in translational science and the grey area of academic. Rev. Esp. Pedag. 2018, 76, 413-435. [CrossRef] 
3. Orcos, L.; Hernández-Carrera, R.M.; Espigares, M.J.; Magreñán, Á.A. The Kumon Method: Its Importance in the Improvement on the Teaching and Learning of Mathematics from the First Levels of Early Childhood and Primary Education. Mathematics 2019, 7, 109. [CrossRef]

4. Orcos, L.; Jordán, C.; Magreñán, A.A. 3D visualization through the hologram for the learning of area and volume concepts. Mathematics 2019, 7, 247. [CrossRef]

5. Orcos, L.; Arias, R.; Magreñán, A.A.; Sicilia, J.A.; Sarría, Í. Computer Application for the Evaluation of Mathematical Competence in Secondary Education: A Case Study. Commun. Comput. Inf. Sci. 2019, 1011, 162-173.

6. Orcos, L.; Magreñán, Á.A. The hologram as a teaching medium for the acquisition of STEM contents. Int. J. Learn. Tech. 2018, 13, 163-177. [CrossRef]

7. Sarría, Í.; González, R.; Magreñán, Á.A.; Narváez, S.P.; Orcos, L. Games math. Adaptive video game to evaluate basic mathematic concepts. Commun. Comput. Inf. Sci. 2017, 734, 27-35.

8. Hörmander, L. An Introduction to Complex Analysis in Several Variables; North-Holland Publishing Company: Amsterdam, The Netherlands, 1973.

9. Shabat, B.V. Introduction to Complex Analysis PART II, Functions of Several Variables; American Mathematical Society: Providence, RI, USA, 1992.

10. Traub, J.F. Iterative Methods for the Solution of Equations; Prentice-Hall: Englewood Cliffs, NJ, USA, 1964.

11. Kung, H.T.; Traub, J.F. Optimal order of one-point and multipoint iteration. J. Assoc. Comput. Mach. 1974, 21, 643-651. [CrossRef]

12. Ostrowski, A.M. Solution of Equations in Euclidean and Banach Space; Academic Press: New York, NY, USA, 1973.

13. Dong, C. A basic theorem of constructing an iterative formula of the higher order for computing multiple roots of an equation. Math. Numer. Sin. 1982, 11, 445-450.

14. Dong, C. A family of multipoint iterative functions for finding multiple roots of equations. Int. J. Comput. Math. 1987, 21, 363-367. [CrossRef]

15. Kumar, S.; Kanwar, V.; Singh, S. On some modified families of multipoint iterative methods for multiple roots of nonlinear equations. Appl. Math. Comput. 2012, 218, 7382-7394. [CrossRef]

16. Victory, H.D.; Neta, B. A higher order method for multiple zeros of nonlinear functions. Int. J. Comput. Math. 1983, 12, 329-335. [CrossRef]

17. Neta, B.; Jhonson, A.N. High order nonlinear solver for multiple roots. Comput. Math. Appl. 2008, 55, 2012-2017. [CrossRef]

18. Neta, B. New third order nonlinear solvers for multiple roots. Appl. Math. Comput. 2008, 202, $162-170$. [CrossRef]

19. Neta, B. Extension of Murakami's high order nonlinear solver to multiple roots. Int. J. Comput. Math. 2010, 87, 1023-1031. [CrossRef]

20. Li, S.G.; Cheng, L.Z.; Neeta, B. Some fourth-order nonlinear solvers with closed formulae for multiple roots. Comput. Math. Appl. 2010, 59, 126-135. [CrossRef]

21. Li, S.; Liao, X.; Cheng, L. A new fourth-order iterative method for finding multiple roots of nonlinear equations. Appl. Math. Comput. 2009, 215, 1288-1292.

22. Jarratt, P. Multipoint iterative methods for solving certain equations. Comput. J. 1966, 8, 398-400. [CrossRef]

23. Sharma, J.R.; Sharma, R. Modified Jarratt method for computing multiple roots. Appl. Math. Comput. 2010, 217, 878-881. [CrossRef]

24. Zhou, X.; Chen, X.; Song, Y. Constructing higher-order methods for obtaining the multiple roots of nonlinear equations. J. Comput. Appl. Math. 2011, 235, 4199-4206. [CrossRef]

25. Ahlfors, L.V. Complex Analysis; McGraw-Hill Book, Inc.: New York, NY, USA, 1979.

26. Geum, Y.H.; Kim, Y.I.; Neta, B. Constructing a family of optimal eighth-order modified Newton-type multiple-zero finders along with the dynamics behind their purely imaginary extraneous fixed points. J. Comput. Appl. Math. 2018, 333, 131-156. [CrossRef]

(C) 2019 by the authors. Licensee MDPI, Basel, Switzerland. This article is an open access article distributed under the terms and conditions of the Creative Commons Attribution (CC BY) license (http:/ / creativecommons.org/licenses/by/4.0/). 